\title{
Purine and pyrimidine phosphoribosyltransferases: A versatile tool for enzymatic synthesis of nucleoside-5'-monophosphates
}

Del Arco, Jon; Fernandez Lucas, Jesus

\begin{abstract}
Background: In recent years, enzymatic methods have shown to be an efficient and sustainable alternative for the synthesis of nucleosides and nucleoside-5'monophosphates (NMPs) to the traditional multistep chemical methods, since chemical glycosylation reactions include several protection-deprotection steps and the use of chemical reagents and organic solvents that are expensive and environmentally harmful. Results: In this mini-review, we want to illustrate the application of phosphoribosyltransferases (PRTs) in enzymatic synthesis of NMPs. In this sense, many different examples about the use of PRTs as biocatalysts, as whole cells or enzymes, are described. In addition, it also includes detailed comments about structure and catalytic mechanism of described PRTs, as well as their possible biological role and therapeutic use, substrate specificity and advances in detection of new enzyme specificities towards different substrates. In addition, several examples about the use of PRTs in mono or multi-enzymatic synthesis of NMP analogues are shown. Finally, a brief discussion about advantages and drawbacks of the use of PRTs as industrial biocatalyst of NMPs has been commented. Conclusion: Despite the great potential of PRTs as biocatalysts for industrial synthesis of NMPs, several drawbacks must be overcome before reaching a suitable industrial application. In this sense, multienzyme systems provide an appropriate framework for this purpose. Moreover, future advances in different disciplines as protein engineering, bioinformatics and omics will help to reach this goal.
\end{abstract}

\section{Keywords}

Biocatalysis, Nucleic acid derivatives, Nucleoside-5'-monophosphates (NMPs), NucleosidesPhosphoribosyltransferase 